\title{
Lack of Clinical Trial Data Transparency and Current Solutions
}

\author{
Tabassom Baghai, BSc ${ }^{1}$ \\ ${ }^{1}$ Department of Biochemistry, Microbiology and Immunology, Faculty of Medicine, University of Ottawa
}

\section{A B STRACT}

An ongoing challenge in clinical research is the inaccessibility of clinical trial data, which prevents physicians from making an informed decision with regards to patient care. The U.S. Food and Drug Administration (FDA) as well as the World Health Organization (WHO) recently called for all trial data to be registered and made publically available. However, this issue is still ongoing and there are several measures currently being enforced to rectify these concerns. Potential solutions, such as regulations, campaigns, and possible consequences, for increasing transparency in clinical trial data will be discussed.

\section{RÉSUMÉ}

L'inaccessibilité des données provenant d'essais cliniques constitue un défi constant en recherche clinique, puisqu'elle empêche les médecins de prendre des décisions éclairées quant aux soins de leurs patients. Récemment, le Secrétariat américain aux produits alimentaires et pharmaceutiques (FDA) ainsi que l'Organisation mondiale de la Santé (OMS) ont demandé que toutes les données d'essais cliniques soient enregistrées et mises à la disposition du public. Toutefois, ce problème persiste et plusieurs mesures ont été mises en place pour répondre à ces préoccupations. Des solutions possibles dont des réglementations, des campagnes et des sanctions possibles pour améliorer la transparence en ce qui concerne les données d'essais cliniques seront discutées.

\section{INTRODUCTION}

Treatment of patients using drugs or devices is based on the implicit trust that physicians are making rational decisions founded upon thorough knowledge of the efficacy and safety of the treatment. Once drugs and devices are developed, they are required to undergo various trial phases to test their effects on human participants who have been deemed appropriate for the study [1]. The main objective of these trials is to identify potential benefits as well as the harmful and adverse effects of these treatments [1]. Therefore, it is imperative that these results are accessible to physicians so they may determine the best treatment options for their patients. However, a current issue in clinical research is the absence of data obtained through these trials, thus limiting the basis on which physicians make their decisions [2]. This has caused the safety of patients to be at risk and will continue doing so unless the proper steps and regulations are implemented [2].

\section{SELECTIVE REPORTING AND PATIENT SAFETY}

Selective reporting, in this setting, can be defined as the act of excluding data obtained from clinical trials [3]. This can occur due to various reasons, including results that are deemed null or negative [4], or in the case of industry-funded research, treatment that does not display the desired effects the study's sponsor had hoped for [5]. Therefore, the data obtained is revised for commercial purposes so it may appear more promising [5]. This phenomenon is known as reporting bias, where researchers are more likely to report positive data as opposed to negative data
[6]. This leads the public, including health care practitioners, to be misinformed and unintentionally develop a skewed opinion of the treatment [2]. As a result, treatments become overvalued and their harmful effects become underestimated, leading patients to potentially be exposed to toxicities and adverse events in the absence of any clinical benefits [3]. Moreover, without an in-depth understanding of the treatment in question, it is impossible for physicians to make a proper comparison between various treatments [2]. Consequently, a physician may select a promising drug based on the presented data, as opposed to another drug that would have been a safer choice [2]. A well-documented example that highlights the consequences of selective reporting is rofecoxib (Vioxx), a COX-2 inhibitor [3]. The risks associated with rofecoxib were not properly represented in the trial reports, which falsely reported rofecoxib's benefits on the gastrointestinal system as greater than its risks on the cardiovascular system [7]. This resulted in 88,000 to 140,000 serious cases of heart disease in the United States alone, thus resulting in the removal of rofecoxib from the market in 2004 [8].

Furthermore, without a clear representation of clinical trial results, selective reporting could lead other investigators to conduct similar trials exposing patients to adverse effects that may have been prevented with clear communication [2]. Not only does this put more patients at harm's risk, it wastes time and money that could have been spent on alternative treatments [2]. 


\section{CURRENT APPROACHES}

The World Health Organization (WHO) recently issued a statement advocating for the release of all data obtained from clinical trials, including those from prior years [9]. Moreover, they have also stated that the results obtained should be submitted for publication in a journal within 12 months of study completion or made publicly accessible within 24 months of study completion [10]. The availability of this knowledge will facilitate rational decision-making that is based on the safety and efficacy of treatments while avoiding misinformation and unwarranted costs [3]. WHO's statement is in support of various policies and campaigns currently taking place, which will be thoroughly discussed below.

\section{Trial Registration}

In 2007, the Food and Drug Administration Amendments Act (FDAAA) was implemented [11]. The FDAAA required all clinical trials and collected data to be registered on ClinicalTrials.gov [11]. ClinicalTrials.gov is currently the largest clinical trial database with over 200,000 trial registrations globally [12]. However, even with an accessible registry and the implemented rule of registering clinical trials, a large number of trials are continuing to omit data [13]. The FDAAA attempted to enforce the reporting of trial results with a penalty of up to $\$ 10,000$ per day if they were not reported within twelve months of completion [14]. However, disadvantages of the ruling included the focus on ongoing trials in the United States as well as neglecting to enforce the penalty $[6,14]$. This led to many trials, including those registered, to continue to withhold results [14]. Additionally, the FDAAA ruling excludes clinical trial results obtained prior to 2007 [15], thus limiting a relatively large volume of data of which many treatments are based on today [6].

The International Committee of Medical Journal Editors (ICMJE) is attempting to help this ongoing problem by suggesting that a plan for sharing trial data should also be included with the registration [16]. They have also implemented the rule that its journals will only publish clinical trials that have been registered prior to the start of data collection [16]. The enforcement of this rule has been inconsistent and flawed, since it primarily affects clinical trials whose goal is to be published [6].

Recently, an online tool known as TrialsTracker was developed to track registered trials that have not made their results available [17]. A study looking at trials registered between 2006 and 2014, determined that $45 \%$ of registered trials were missing data associated with their study [18]. Furthermore, among the top 100 universities and institutions that were associated with a large number of absent results, nine were identified to be Canadian [18]. Interestingly, the Ottawa Hospital Research Institute (OHRI) was ranked 99 among the institutions mentioned, with $63.8 \%$ of its trials missing results [18]. This suggests that stronger rules are required to ensure that all trial data is made available for health care practitioners and patients. Registration has been a good step towards informing the public of current clinical trials [19]. However, it is the results obtained from the trials that will help determine future decisions and outcomes of patient care and safety [19]. Not only would transparency of the results help with better decision-making, it will also help facilitate the recruitment of more patients, as patients will be better aware of studies that are being conducted [20]. In addition, transparency will aid investigators, as it will prevent the unnecessary duplications of studies [2]. Moreover, having the option to share results in a clinical trial database could help avoid reporting bias since it would allow all data to be available and not only those deemed positive [2].

\section{AllTrials Campaign}

In 2013, the AllTrials campaign began in order to improve the registration and sharing of the methods and results to the public [21]. This campaign believes that all clinical trials should be registered and results made available [21]. The AllTrials campaign has received support from a number of organizations as well as patients, physicians, and investigators around the world [21]. Thus far, they have been successful in raising awareness using petitions that they forward to health and policy regulators in various countries [21]. Having its members write to individuals involved with the regulation of clinical trials, especially Members of the Parliament in Europe and Canada, has led to the passing of laws that enforce the availability of clinical trial data [22]. Furthermore, there is an increase in the number of pharmaceutical companies that are joining the AllTrials campaign, including GlaxoSmithKline (GSK) [22]. Interestingly, GSK pleaded guilty in failing to report safety data regarding Avandia, a drug used for diabetes as well as misbranding antidepressants, Paxil and Wellbutrin, in 2012, which resulted in GSK paying \$3 billion in fines [23]. Thus, joining this campaign could help them repair their public credibility.

\section{FUTURE PLANS}

\section{Audits}

Many organizations and investigators continue to avoid publishing their trial data regardless of the regulations that were implemented [19]. For this reason, it may prove useful to conduct audits of the registered trials and publically identify those who have omitted data in addition to those who have been updating their database [19]. TrialsTracker is an example of an audit tool that has identified trials whose results have not been available [17]. Further audits could potentially lead investigators and companies to be accountable and begin adhering to regulations [19]. Through these audits, health care practitioners will be able to make improved and informed decisions, as they will be aware of 
credible results [19].

\section{Data-sharing Platform}

Another option is to implement a data-sharing platform that will help deliver trial data in a responsible and unbiased manner [16]. An example of such a platform is the Yale Open Data Access (YODA) Project, which first started in 2011 [16]. The YODA Project acts as an independent third party intermediary between organizations and researchers that perform clinical trials and those interested in the trial results [16]. They act to objectively evaluate the data obtained in order to provide important and crucial information about treatments that aid in patient care decisions [16]. This model system could potentially help to increase trust in the data obtained from clinical trials. Johnson \& Johnson, a large healthcare company, has recently partnered with the YODA project for the release of their clinical trial data [16]. Although journal publications might not be ideal for all results obtained from clinical trials, having a platform to share and report information is a proper stepping-stone for improving patient safety.

\section{POTENTIAL CONSEQUENCES OF MANDATORY REPORTING}

As mentioned thus far, the transparency of clinical trial data has become a hot topic of discussion in healthcare. Emphasis has been on the importance and benefits of having data available in order to increase the quality of patient health and safety [24]. The potential benefits that could emerge from proper access and availability of clinical trial data are undeniable; however, the consequences that could arise as a result should be taken into account. One issue is that transparency of data could jeopardize patient confidentiality, as the details entailed for each patient in clinical trials could potentially expose their identities [24]. Appropriate measures for data de-identification must be taken and controlled access to the data should be implemented in order to ensure patient privacy remains intact [24].

A higher level of transparency could also put commercial confidentiality at risk, especially when it comes to the pharmaceutical industry [25]. Many companies will become wary that their trade secrets and proprietary information could be made public, thus continuing to affect their willingness to share data as it may hinder product research and development [25]. Moreover, the availability of clinical trial information may also hinder the incentive for obtaining patents for drugs when properties and effects have already been disclosed [25]. For instance, there is currently a lot of focus on developing novel uses for drugs that were initially developed and tested for a certain disease, but instead possess the potential to be an effective treatment for another disease [25]. By enforcing complete transparency of data, its use as a treatment for the other disease may no longer seem novel enough for a patent, thus discouraging researchers from developing drugs through this approach [25]. Interferon- $\alpha$ is an example of a drug that was initially developed for the treatment of hairy-cell leukemia [26]. It is currently used for the treatments of hepatitis $C$ as well as metastatic melanoma, among other diseases [26].

Another aspect that should be considered is whether or not to limit access to information obtained from a clinical trial to authorized researchers only [6]. It may be beneficial for the data to be independently analyzed by other researchers, especially through the emerging data-sharing platforms, in order to gain other perspectives [6]. However, if the results fall into the wrong hands or are analyzed in an unethical manner, it could lead to the disclosure of misleading information to the public, evoking unnecessary treatments due to unwarranted health scares [6].

\section{CONCLUSION}

The inaccessibility of clinical trial data remains an ongoing concern in clinical research [2]. In many cases, this has resulted in harm or even death of patients whose treatment was based on the trial data available to physicians [3]. To solve this issue, registration of clinical trials is being implemented [12], while an international campaign, AllTrials, is calling out for the release of necessary trial data, past and present [21]. Furthermore, audits are recommended as a way to specifically identify unreliable trials [19]. Data-sharing platforms are produced to present an unbiased and responsible distribution of trial data [16]. The incorporation of publication officers in the organization is taking place at the OHRI to assist with reporting clinical trial results [18]. Their role is to direct the researchers at The Ottawa Hospital in properly preparing and submitting their results for publication [18]. Making clinical trial data more accessible must continue to be implemented as it can affect a large number of people, including patients and health care practitioners. However, its risks must also be taken into account when developing policies and approaches. It is imperative that health-related research remains focused on what is best for patients.

\section{REFERENCES}

1. Umscheid CA, Margolis DJ, Grossman CE. Key Concepts of Clinical Trials: A Narrative Review. Postgrad Med. 2011;123(5):194-204.

2. Brassington I. The ethics of reporting all the results of clinical trials. Br Med Bull. 2017;121(1)19-29.

3. Gøtzsche PC. Why we need easy access to all data from all clinical trials and how to accomplish it. Trials. 2011;12(1):249.

4. Dickersin K, Chalmers I. Recognizing, investigating and dealing with incomplete and biased reporting of clinical research: from Francis Bacon to the WHO. J R Soc Med. 2011;104(12):532-8.

5. Wynia $M$, Boren $\mathrm{D}$. Better regulation of industry-sponsored clinical trials is long overdue. J Law Med Ethics. 2009;37(3):410-9.

6. Richardson E. Health Policy Brief: Transparency in Clinical Research [Internet]. Health Affairs. 2016 Jan 14. Available from: http://healthaffairs.org/ healthpolicybriefs/brief_pdfs/healthpolicybrief_150.pdf

7. Krumholz HM, Ross JS, Presler AH, et al. What have we learnt from Vioxx? BMJ. 2007;334(7585):120-3.

8. Madigan D, Sigelman DW, Mayer JW, et al. Under-reporting of cardio- 
vascular events in the rofecoxib Alzheimer disease studies. Am Heart J. 2004;164(2):186-93.

9. Jasarevic T. WHO call for increased transparency in medical research [Internet]. Geneva: World Health Organization; 2015 Apr 14. Available from: http://www.who.int/mediacentre/news/notes/2015/medical-researchtransparency/en/

10. WHO Statement on Public Disclosure of Clinical Trial Results [Internet]. World Health Organization; 2015 Apr 9. Available from: http://www.who. int/ictrp/results/reporting/en/

11. Food and Drug Administration Amendments Act of 2007 [Internet]. U.S Public Law 110-85. 2007 Sept 27. Available from: http://www.gpo.gov/fdsys/ pkg/PLAW-110publ85/pdf/PLAW-110publ85.pdf

12. Trends, Charts, and Maps [Internet]. Clinicaltrials.gov, U.S. National Institutes of Health. 2016 Feb. Available from: https://clinicaltrials.gov/ct2/resources/trends

13. Jones CW, Handler L, Crowell KE, et al. Non-publication of large randomized clinical trials: cross sectional analysis. BMJ. 2013;347:f6104.

14. Miller JE, Korn D, Ross JS. Clinical trial registration, reporting, publication and FDAAA compliance: a cross-sectional analysis and ranking of new drugs approved by the FDA in 2012. BMJ Open. 2015;5(11):e009758.

15. FDAAA 801 Requirements [Internet]. Clinicaltrials.gov, U.S. National Institutes of Health. 2017 Feb. Available from: https://clinicaltrials.gov/ct2/ manage-recs/fdaaa

16. Krumholz, HM. The Yale Open Data Access (YODA) Project - A Mechanism for Data Sharing. N Engl J Med. 2016;375:403-5.

17. Powell-Smith A, Goldacre B. The TrialsTracker: Automated ongoing monitoring of failure to share clinical trial results by all major companies and research institutions. F1000Research. 2016;5:2629.

18. Hol D. 'We've been deceived': Many clinical trial results are never published [Internet]. CBC News, Canada; 2016 Nov 24. Available from: http://www. cbc.ca/news/health/clinical-trial-results-not-published-1.3865785

19. Goldacre B. How to Get All Trials Reported: Audit, Better Data, and Individual Accountability. PLOS Medicine. 2015;12(4):e1001821.

20. Ghersi D, Clarke M, Berlin J, et al. Reporting the findings of clinical trials: a discussion paper [Internet]. Bulletin of the World Health Organization. 2008;86(6):417-96.

21. AllTrials [Internet]. London, UK. 2013 Sept. Available from: http://www.alltrials.net/find-out-more/all-trials/

22. Lane, S. AllTrials campaign and petition support greater clinical trial transparency. [Internet] Elsevier. 2014 June 23. Available from: https://www. elsevier.com/connect/alltrials-campaign-supports-greater-clinical-trialtransparency

23. Thomas K, Schmidt MS. Glaxo Agrees to Pay \$3 Billion in Fraud Settlement. [Internet] The New York Times. 2012 July 2. Available from: http://www.nytimes.com/2012/07/03/business/glaxosmithkline-agrees-to-pay-3-billionin-fraud-settlement.html

24. Tucker $\mathrm{K}$, Branson J, Dilleen $\mathrm{M}$, et al. Protecting patient privacy when sharing patient-level data from clinical trials. BMC Med Res Methodol. 2016;16(1):77.

25. Price WN, Minssen T. Will clinical trial data disclosure reduce incentives to develop new uses of drugs? Nat Publ Gr. 2015;33(7):685-6.

26. Roin BN. Solving the problem of new uses [Internet]. Social Science Research Network. 2013 October 10. Available from: http:// dx.doi.org/10.2139/ ssrn. 2337821 